Check for updates

Cite this: Chem. Commun., 2021, 57,9582

Received 17th June 2021,

Accepted 12th August 2021

DOI: $10.1039 / \mathrm{d} 1 \mathrm{cc} 03221 \mathrm{f}$

rsc.li/chemcomm

\section{Cavity-promotion by pillar[5]arenes expedites organic photoredox-catalysed reductive dehalogenations $\dagger$}

\author{
Maximilian Schmidt (D) a and Birgit Esser (ID *abc
}

The efficiency of the photo-induced electron transfer in photoredox catalysis is limited by the diffusional collision of the excited catalyst and the substrate. We herein present cavity-bound photoredox catalysts, which preassociate the substrates, leading to significantly shortened reaction times. A pillar[5]arene serves as the cavity and phenothiazine as a catalyst in the reductive dehalogenation of aliphatic bromides as a proof of concept reaction.

Photoredox catalysts (PRCs) allow the conversion of light into chemical energy. ${ }^{1,2}$ They are considered a more sustainable alternative to traditional catalyst systems, using light as a nonhazardous and environmentally friendly energy source instead of high temperatures or harsh reaction conditions. Significant advances have been achieved in recent years using PRCs in synthetic organic chemistry. ${ }^{3-5}$ Both transition metal complexes $^{6,7}$ as well as organic dyes ${ }^{8-10}$ have successfully been employed in a variety of synthetic transformations. One of the critical steps in photoredox catalysis is the photo-induced electron transfer (PET) from the catalyst in its excited state to the substrate, which usually is the first step of the catalytic cycle. The efficiency of the PET is limited by the diffusional collision of the catalyst and substrate during the lifetime of the excited state of the catalyst. ${ }^{11}$ This leads to photoredox reactions often suffering from long reaction times, thereby consuming high amounts of light energy, or high catalyst loadings being necessary. ${ }^{1}$ We herein present a catalyst system, where

\footnotetext{
${ }^{a}$ Institute for Organic Chemistry, University of Freiburg, Albertstraße 21, 79104 Freiburg, Germany. E-mail: besser@oc.uni-freiburg.de www.esser-lab.uni-freiburg.de

${ }^{b}$ Freiburg Materials Research Center, University of Freiburg, Stefan-Meier-Str. 21, 79104 Freiburg, Germany

${ }^{c}$ Freiburg Center for Interactive Materials and Bioinspired Technologies, University of Freiburg, Georges-Köhler-Allee 105, 79110 Freiburg, Germany $\dagger$ Electronic supplementary information (ESI) available: Materials and methods, synthetic manipulations, NMR spectra, cyclic voltammograms, absorption and emission spectra, determination of excited state reduction potentials, determination of photon flux of the nail dryer lamp and details of DFT calculations. See DOI: $10.1039 / \mathrm{d} 1 \mathrm{cc} 03221 \mathrm{f}$
}

the PRC is covalently bound to a cavity. This cavity can preassociate the substrate to make the PET more efficient by holding the substrate and catalyst in close proximity (Fig. 1A). ${ }^{12}$ After completion of the PR catalytic cycle, the catalyst cavity is free to associate with a new substrate. Our catalyst design uses a pillar[5] arene (PA) as the cavity and phenothiazine (PT) as the PRC (Fig. 1B). Pillarenes have become versatile supramolecular hosts due to their facile synthesis, unique pillar shape, the possibility for further functionalization and their host-guest properties. ${ }^{13,14}$ They have been used in supramolecular catalysis on several occasions ${ }^{15}$ as well as in photocatalysis. ${ }^{16}$ While

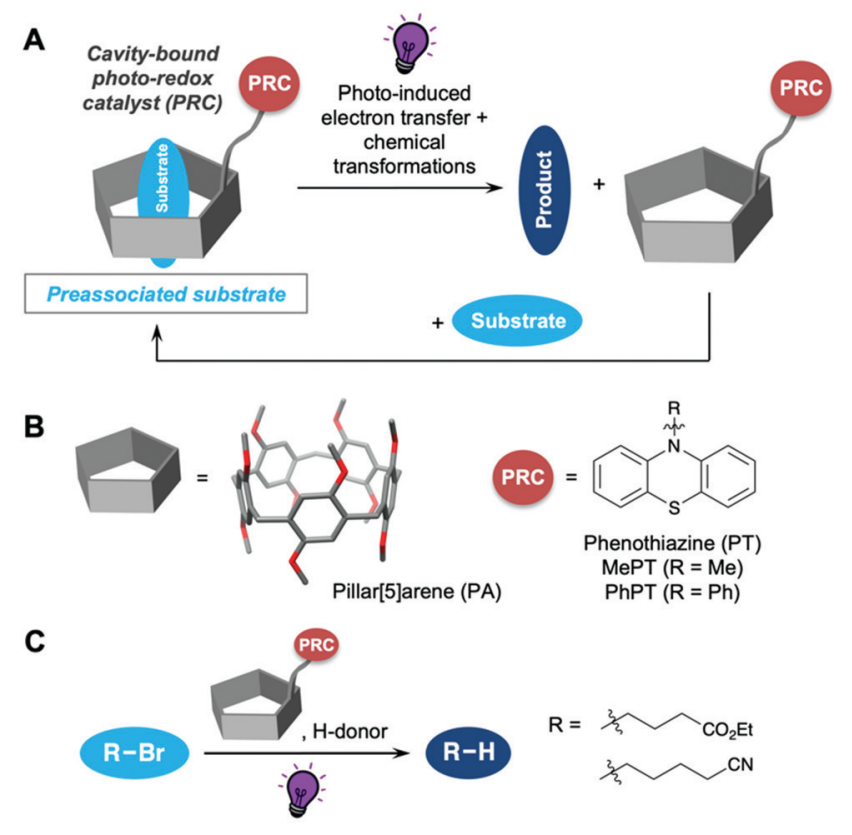

Fig. 1 (A) Concept of cavity-promotion in photoredox catalysis, investigated herein; (B) structure of pillar[5]arene (PA), used as a cavity, and phenothiazine (PT) as a photoredox catalyst; (C) photoredox-catalyzed reductive dehalogenation of aliphatic bromides used as a proof-ofconcept reaction in this study. 
PET to substrates bound by a cage has been reported on a few occasions before, ${ }^{17-20}$ no application of a pillarene in photoredox catalysis has yet appeared to the best of our knowledge.

As a proof of concept reaction we chose the reductive dehalogenation of aliphatic bromides (Fig. 1C). This reaction, developed by Hawker and Read de Alaniz in $2015,{ }^{21}$ allows for a mild photocatalytic dehalogenation using $N$-phenyl-PT (PhPT) as the PRC instead of the traditional protocols involving metalhalogen exchange, ${ }^{2,23}$ tin hydrides ${ }^{24}$ or other highly toxic reagents. $^{25-27}$ We used a UV nail dryer lamp as a widely available and cheap light source in this reaction. In spite of its lower light intensity compared to typically used LED setups, we were able to obtain good yields for the reductive dehalogenations, since cavity promotion increased the efficiency of the PET. This is an advantage in comparison to many PRC studies, which use specialized equipment and radiation sources not available to a standard synthetic laboratory. PT derivatives have also been employed in metal-free atom-transfer radical polymerizations $^{28-30}$ as well as many other reaction types. ${ }^{31,32}$ Due to their reversible redox chemistry, phenothiazine derivatives have also found use as organic battery materials ${ }^{33-35}$ and in other supramolecular applications. ${ }^{36}$

We designed three cavity-linked catalysts PA-PhPT, PA-C4-PhPT and PA-C4-PT (Scheme 1), which differ in the choice of linker between the pillar[5]arene and phenothiazine. The synthesis of all three started from decamethoxy-pillar[5]arene (1), ${ }^{37}$ which we

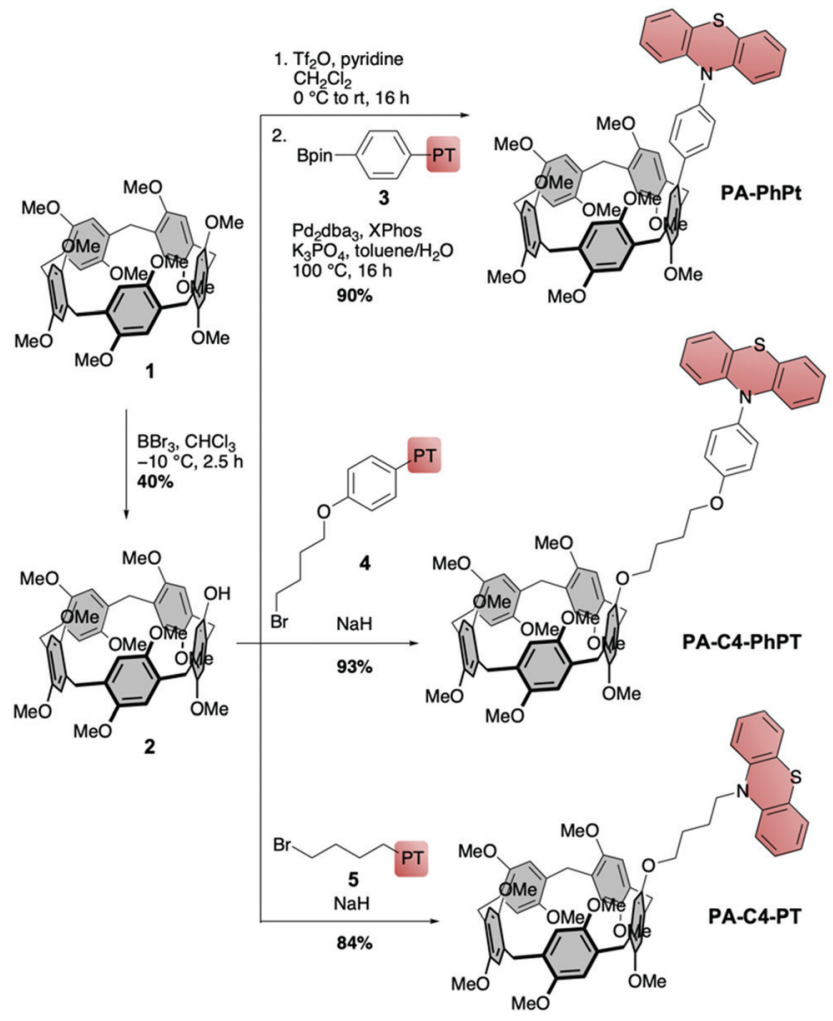

Scheme 1 Synthesis of cavity-linked catalysts with pillar[5]arene as the cavity and phenothiazine as a photoredox catalyst (with excited state reduction potentials vs. SCE). transformed into mono-hydroxy-PA 2 following literature procedures. $^{38,39}$ Transformation of the hydroxy to a triflate group followed by Suzuki-Miyaura coupling with PT-based boronic ester 3 led to PA-PhPT in a high yield of $90 \%$ over these two steps. For butyl-linked PA-C4-PhPT we reacted 2 with PT-functionalized bromide 4, and nucleophilic substitution furnished the catalyst in excellent yield of $93 \%$. The same procedure using bromide 5 afforded catalyst PA-C4-PT in a high yield of $84 \%$. For the syntheses of PT precursors 3-5, see the ESI. $\dagger$ The PA-linked PRCs were characterized using 1 - and $2 \mathrm{D}{ }^{1} \mathrm{H}$ - and ${ }^{13} \mathrm{C}$ NMR spectroscopy and HRMS. We determined their excited state reduction potentials using cyclic voltammetry, UV/Vis absorption and emission spectroscopy (for data see the ESI $\dagger$ ). ${ }^{8}$ With $E_{1 / 2}{ }^{*}=-2.84 \mathrm{~V}$ (PA-PhPT), $-2.85 \mathrm{~V}$ (PA-C4-PhPT) and $-2.80 \mathrm{~V}$ (PA-C4-PT), the cavity-linked phenothiazines have slightly stronger reducing abilities compared to $\mathrm{PhPT}\left(E_{1 / 2}{ }^{*}=-2.56 \mathrm{~V}\right)$ and $\mathrm{MePT}\left(E_{1 / 2}^{*}=-2.69 \mathrm{~V}\right.$, all $v s$. SCE). Pillarene 1 has no significant absorption at the wavelength used in the reaction (emission maximum of the nail dryer lamp at $365 \mathrm{~nm}$ ), and hence no excited state of $\mathbf{1}$ is formed that could interfere with the mechanism.

We next employed the cavity-bound PRCs PA-PhPT, PA-C4-PhPT and PA-C4-PT in the reductive dehalogenation of ethyl 4-bromobutanoate (6) and 5-bromopentanenitrile (7) and compared the results to PhPT and MePT (see Fig. 1B) under identical conditions. We used $5 \mathrm{~mol} \%$ of the PRC and five equivalents each of diisopropylethylamine and formic acid in acetonitrile (Table 1). ${ }^{21}$ As a light source we used a cheap and widely available nail dryer lamp with an emission maximum of the bulb at $365 \mathrm{~nm}$ (for experimental setup see the ESI $\dagger$ ). This lamp has a lower photon flux compared to typical LED setups used in the literature, which we determined using ferrioxalate actinometry ${ }^{40}$ as a simple yet accurate method (see the $\mathrm{ESI} \dagger$ for details and comparative values). Hence for the debromination of 6 and 7 using PhPT as PRC, the yield within $48 \mathrm{~h}$ amounted to $29 \%$ and $28 \%$ (entry 1), respectively, while $81 \%$ was obtained after $24 \mathrm{~h}$ for 6 in the literature using the higher flux LED setup. Using MePT we obtained 17\% and 16\%

Table 1 Comparison of cavity- and non-cavity-bound phenothiazine photoredox catalysts (PRCs) in the reductive dehalogenation of alkyl bromides 6 and 7

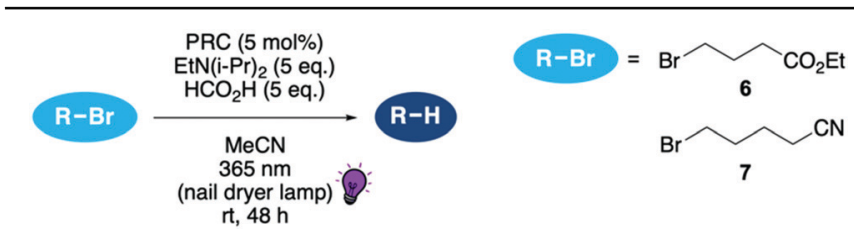

\begin{tabular}{llll}
\hline Entry & Catalyst (PRC) & ${\text { Yield }(\mathrm{R}-\mathrm{Br}=\mathbf{6})^{a}}^{a}$ & ${\text { Yield }(\mathrm{R}-\mathrm{Br}=7)^{a}}^{a}$ \\
\hline 1 & PhPT & $29 \%$ & $28 \%$ \\
2 & MePT & $17 \%$ & $16 \%$ \\
3 & MePT + 1 & $17 \%$ & $14 \%$ \\
4 & 1 & $0 \%$ & $0 \%$ \\
5 & PA-PhPT & $55 \%$ & $61 \%$ \\
6 & PA-C4-PhPT & $61 \%$ & $63 \%$ \\
7 & PA-C4-PT & $49 \%$ & $60 \%$
\end{tabular}

${ }^{a}$ Conversions after $48 \mathrm{~h}$ as the mean of three reactions, determined by ${ }^{1} \mathrm{H}$ NMR spectroscopy. 
yield, respectively, using our setup due to the lower stability of its excited and/or oxidized state compared to $\mathrm{PhPT}^{28}$ (entry 2). Simply adding decamethoxypillar[5]arene (1) to the reaction with MePT did not expedite its course (entry 3), and the presence of sole pillarene 1 did not lead to any transformation (entry 4). Using the cavitybound PRCs PA-PhPT, PA-C4-PhPT and PA-C4-PT, on the other hand, led to significant increases in yield by factors of 1.6-3.8 compared to PhPT and MePT (entries 5-7). A particularly large improvement is observed for PA-C4-PT with 2.9 and 3.8 times higher yields, respectively, compared to MePT (entry 6); however, this catalyst was slightly less stable towards decomposition compared to the other catalysts due to the alkyl substitution on the phenothiazine nitrogen atom. The best overall performance was observed for PA-C4-PhPT and PA-PhPT with aryl substituents on the phenothiazine nitrogen atoms. Of these two, PA-PhPT is easier to synthesize in a gram scale, since no column chromatography is necessary in the purification of the PT-precursor, and this catalyst could also be recovered quantitatively after each photoredoxcatalysed transformation. The increase in reaction rate can also be seen from the kinetic profiles comparing the rates of the dehalogenation of bromopentanenitrile (7) with PA-PhPT and PhPT (see the ESI $\dagger$ ).

The results of the photoredox-catalysed experiments demonstrate that the pillarene cavity efficiently expedites the reductive dehalogenation reaction by keeping the substrate in close proximity to the catalyst. This is supported by the known ability of pillar[5]arenes to bind electron-poor alkyl halides. ${ }^{14}$ The association constant for the complexation of 5-bromopentanenitrile (7) by 1 amounts to $1.7 \times 10^{4} \mathrm{M}^{-1}{ }^{41}$ This is 30 times higher than that for the debrominated product of the dehalogenation reaction, pentanenitrile, which has an association constant of $5.7 \times 10^{2} \mathrm{M}^{-1} \cdot{ }^{41}$ Hence the reduced product will leave the cavity, thereby releasing the catalyst and making room for a new substrate to enter and undergo the reductive dehalogenation. The calculated structure of the complex between PA-PhPT and 5-bromopentanenitrile (7) in Fig. 2 shows its perfect fit into the cavity and the proximity of the bromide to the PT catalyst. This orientation with the bromide of 7 facing toward the PT catalyst is $5.3 \mathrm{kcal} \mathrm{mol}^{-1}$ more stable than the other orientation where the bromide points downward (see the $\mathrm{ESI} \dagger)$.

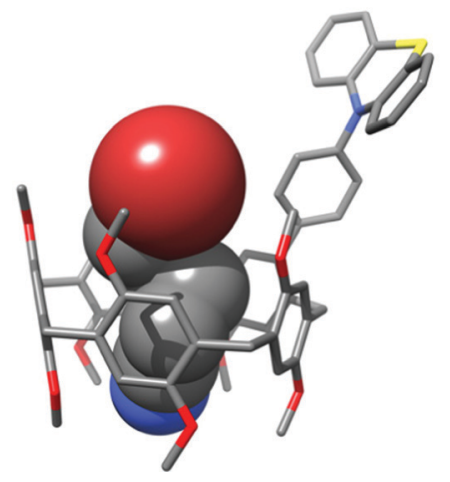

Fig. 2 Calculated structure (PBEh-3c) of the complex between PA-PhPT and 5-bromopentanenitrile (7).
Table 2 Reductive dehalogenations of sterically hindered substrates 8-11 (for reaction conditions see reaction scheme in Table 1) that cannot bind inside the pillar[5]arene cavity using cavity- and non-cavity-bound phenothiazine photoredox catalysts

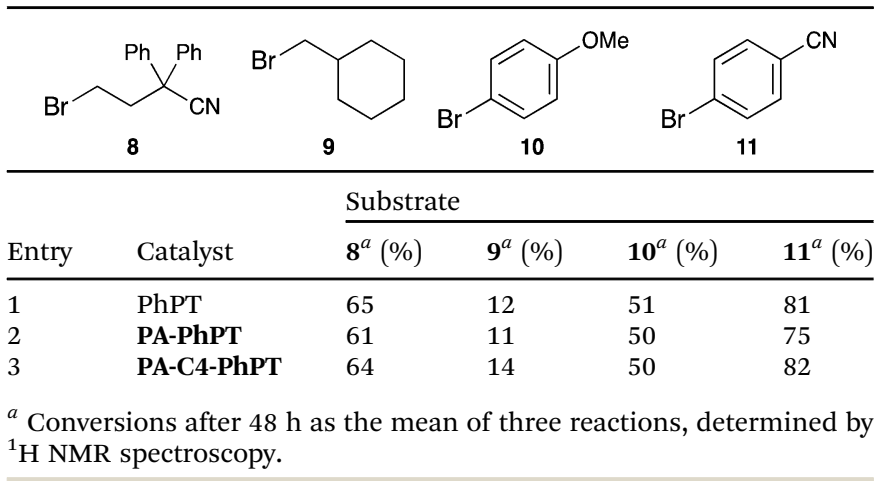

Stern-Volmer fluorescence quenching experiments showed that the excited states of the new catalysts PA-PhPT and PA-C4-PhPT are efficiently quenched by the substrates 6 and 7 (see the ESI $\dagger$ ). Comparison with non-cavity-bound PhPT revealed a more efficient quenching for PA-PhPT and PA-C4-PhPT than for PhPT due to the substrates binding inside the cavities. Pillarene 1, on the other hand, did not quench the fluorescence of PhPT, demonstrating its innocent nature.

To further prove the postulated concept we performed reactions using four substrates that are sterically hindered and cannot bind inside the pillar[5]arene cavity, namely 4-bromo-2,2-diphenylbutanenitrile (8), (bromomethyl)cyclohexane (9), 4-bromoanisole (10) and 4-bromobenzonitrile (11). We used both the pillarene-bound phenothiazine catalysts PA-PhPT and PA-C4-PhPT as well as PhPT as a cavity-free catalyst (Table 2). In each of these cases we obtained a similar yield for the reductive dehalogenation between the three catalysts (compare entries 1-3 for each substrate in Table 2). The slightly lower yields for PA-PhPT can be rationalized by the lower conformational flexibility of the phenothiazine unit due to the rigid phenylene linker to the pillar[5]arene. These comparative experiments demonstrate that when the substrate is unable to bind inside the cavity, the reaction rate remains similar between cavity- and non-cavity bound photoredox catalysts.

In conclusion, we introduced a concept in photoredox catalysis where preassociation of the substrate in close proximity to the catalyst makes the reaction significantly more efficient. We synthesized three pillar[5]arene-functionalized phenothiazines PA-PhPT, PA-C4-PhPT and PA-C4-PT as cavitybound photoredox catalysts and used these in the reductive debromination of ethyl 4-bromobutanoate and 5-bromopentanenitrile. The reactions proceeded with up to 3.8 times higher yield compared with regular phenothiazine catalysts. This concept for photoredox catalysis can be applied to different types and sizes of cavities as well as different catalysts and transformations and will pave the way to making photoredox catalysis more energy- and time-efficient. Furthermore, using 
cavities that selectively bind one substrate over another would allow realizing substrate selectivity.

Generous support by the German Research foundation and the state of Baden-Württemberg through bwHPC is gratefully acknowledged.

\section{Conflicts of interest}

There are no conflicts to declare.

\section{Notes and references}

1 G. E. M. Crisenza and P. Melchiorre, Nat. Commun., 2020, 11, 803.

2 D. M. Schultz and T. P. Yoon, Science, 2014, 343, 1239176.

3 C.-S. Wang, P. H. Dixneuf and J.-F. Soulé, Chem. Rev., 2018, 118, 7532-7585.

4 M. H. Shaw, J. Twilton and D. W. C. C. MacMillan, J. Org. Chem., 2016, 81, 6898-6926.

5 J. P. Barham and B. König, Angew. Chem., Int. Ed., 2020, 59, 11732-11747.

6 C. K. Prier, D. A. Rankic and D. W. C. MacMillan, Chem. Rev., 2013, 113, 5322-5363.

7 J. Twilton, C. Le, P. Zhang, M. H. Shaw, R. W. Evans and D. W. C. MacMillan, Nat. Rev. Chem., 2017, 1, 0052.

8 N. A. Romero and D. A. Nicewicz, Chem. Rev., 2016, 116, 10075-10166.

9 S. G. E. Amos, M. Garreau, L. Buzzetti and J. Waser, Beilstein J. Org. Chem., 2020, 16, 1163-1187.

10 I. K. Sideri, E. Voutyritsa and C. G. Kokotos, Org. Biomol. Chem., 2018, 16, 4596-4614.

11 D. M. Arias-Rotondo and J. K. McCusker, Chem. Soc. Rev., 2016, 45, 5803-5820.

12 C. Yang, T. Mori, T. Wada and Y. Inoue, New J. Chem., 2007, 31, 697.

13 T. Ogoshi, T. Yamagishi and Y. Nakamoto, Chem. Rev., 2016, 116, 7937-8002.

14 Y. Wang, G. Ping and C. Li, Chem. Commun., 2016, 52, 9858-9872.

15 K. Wang, J. H. Jordan, K. Velmurugan, X. Tian, M. Zuo, X. Hu and L. Wang, Angew. Chem., Int. Ed., 2021, 60, 9205-9214.

16 H. Qiang, T. Chen, Z. Wang, W. Li, Y. Guo, J. Yang, X. Jia, H. Yang, W. Hu and K. Wen, Chin. Chem. Lett., 2020, 31, 3225-3229.

17 R. Gera, A. Das, A. Jha and J. Dasgupta, J. Am. Chem. Soc., 2014, 136, 15909-15912.

18 L.-X. Cai, S.-C. Li, D.-N. Yan, L.-P. Zhou, F. Guo and Q.-F. Sun, J. Am. Chem. Soc., 2018, 140, 4869-4876.
19 J. R. Piper, L. Cletheroe, C. G. P. Taylor, A. J. Metherell, J. A. Weinstein, I. V. Sazanovich and M. D. Ward, Chem. Commun., 2017, 53, 408-411.

20 J. S. Train, A. B. Wragg, A. J. Auty, A. J. Metherell, D. Chekulaev, C. G. P. Taylor, S. P. Argent, J. A. Weinstein and M. D. Ward, Inorg. Chem., 2019, 58, 2386-2396.

21 E. H. Discekici, N. J. Treat, S. O. Poelma, K. M. Mattson, Z. M. Hudson, Y. Luo, C. J. Hawker and J. R. de Alaniz, Chem. Commun., 2015, 51, 11705-11708.

22 P. Knochel, W. Dohle, N. Gommermann, F. F. Kneisel, F. Kopp, T. Korn, I. Sapountzis and V. A. Vu, Angew. Chem., Int. Ed., 2003, 42, 4302-4320.

23 W. F. Bailey and J. J. Patricia, J. Organomet. Chem., 1988, 352, 1-46. 24 W. P. Neumann, Synthesis, 1987, 665-683.

25 A. Krief and A.-M. Laval, Chem. Rev., 1999, 99, 745-778.

26 K. Miura, Y. Ichinose, K. Nozaki, K. Fugami, K. Oshima and K. Utimoto, Bull. Chem. Soc. Jpn., 1989, 62, 143-147.

27 M. R. Medeiros, L. N. Schacherer, D. A. Spiegel and J. L. Wood, Org. Lett., 2007, 9, 4427-4429.

28 N. J. Treat, H. Sprafke, J. W. Kramer, P. G. Clark, B. E. Barton, J. Read de Alaniz, B. P. Fors and C. J. Hawker, J. Am. Chem. Soc., 2014, 136, 16096-16101.

29 X. Pan, C. Fang, M. Fantin, N. Malhotra, W. Y. So, L. A. Peteanu, A. A. Isse, A. Gennaro, P. Liu and K. Matyjaszewski, J. Am. Chem. Soc., 2016, 138, 2411-2425.

30 J. P. Cole, C. R. Federico, C. H. Lim and G. M. Miyake, Aldrichimica Acta, 2019, 52, 7-21.

31 D. A. Corbin, C.-H. Lim and G. M. Miyake, Aldrichimica Acta, 2019, 52, 7-21.

32 F. Speck, D. Rombach and H.-A. Wagenknecht, Beilstein J. Org. Chem., 2019, 15, 52-59.

33 F. Otteny, G. Desmaizieres and B. Esser, in Redox Polymers for Energy and Nanomedicine, ed. N. Casado and D. Mecerreyes, Royal Society of Chemistry, 2020, pp. 166-197.

34 B. Esser, Org. Mater., 2019, 01, 063-070.

35 F. Otteny, G. Studer, M. Kolek, P. Bieker, M. Winter and B. Esser, ChemSusChem, 2020, 13, 2232-2238.

36 M. Schmidt, M. Hermann, F. Otteny and B. Esser, Org. Mater., 2020, 02, 235-239.

37 T. Boinski and A. Szumna, Tetrahedron, 2012, 68, 9419-9422.

38 T. Ogoshi, K. Demachi, K. Kitajima and T. Yamagishi, Chem. Commun., 2011, 47, 7164.

39 Y. Chen, M. He, B. Li, L. Wang, H. Meier and D. Cao, RSC Adv., 2013, 3, 21405.

40 C. G. H. Atchard, Proc. R. Soc. London, Ser. A, 1956, 235, 518-536.

$41 \mathrm{X}$. Shu, W. Chen, D. Hou, Q. Meng, R. Zheng and C. Li, Chem. Commun., 2014, 50, 4820-4823. 